\title{
Statistical Entropy of BTZ Black Hole in Higher Curvature Gravity
}

\author{
Hiromi SAIDA ${ }^{a \dagger}$ and Jiro SODA ${ }^{b} \ddagger$ \\ a Graduate School of Human and Environmental Studies, Kyoto University, Kyoto 606-8501, Japan, \\ ${ }^{b}$ Department of Fundamental Sciences, FIHS, Kyoto University, Kyoto 606-8501, Japan
}

\begin{abstract}
For the BTZ black hole in the Einstein gravity, a statistical entropy has been calculated to be equal to the Bekenstein-Hawking entropy. In this paper, the statistical entropy of the BTZ black hole in the higher curvature gravity is calculated and shown to be equal to the one derived by using the Noether charge method. This suggests that the equivalence of the geometrical and statistical entropies of the black hole is retained in the general diffeomorphism invariant theories of gravity. A relation between the cosmic censorship conjecture and the unitarity of the conformal field theory on the boundary of $A d S_{3}$ is also discussed.
\end{abstract}

PACS numbers: 04.70.Dy, 04.50+h, 04.20.Fy

Key Words: Black hole thermodynamics, Diffeomorphism invariant theories of gravity, Statistical entropy, Quantum gravity, AdS/CFT correspondence

\section{INTRODUCTION}

The profound understanding of the black hole thermodynamics is a clue for approaching the quantum gravity. Although the final form of the quantum gravity is covered with a veil of mystery, it would be legitimate to regard the diffeomorphism invariance as the key to the mystery. Hence the black hole thermodynamics in the diffeomorphism invariant theories of gravity should be studied to obtain the insights into the quantum gravity. The first law of black holes in such theories of gravity has already been established [1] [2]. The zeroth and second laws, especially in the higher curvature gravity, have been investigated, for example in ref. [3]. All the black hole entropies treated in the above works are of the integral of geometrical quantities on a spatial section of the event horizon. The geometrical entropy in an N-dimensional higher curvature gravity can be calculated by using the Noether charge method [1] [2] to give

$$
S_{I W}=-\frac{1}{8 G} \oint_{H} d x^{N-2} \sqrt{h} \frac{\partial f}{\partial R_{\mu \nu \alpha \beta}} \epsilon_{\mu \nu} \epsilon_{\alpha \beta},
$$

where $H$ is the spatial section of the event horizon, $h$ is the determinant of the induced metric on $H, f$ is the higher curvature Lagrangian and $\epsilon_{\mu \nu}$ is the binormal to $H$ [2]. When we denote $f=R+$ (higher curvature terms), the first term of this Lagrangian contributes to the entropy (11) as the Bekenstein-Hawking term $A / 4 G$, where $A$ is the area of $H$. The geometrical expression of the black hole entropy obtained in ref. [1] is consistent with the well known results previously obtained for the Einstein gravity, however it does not reveal statistical origin of the entropy. Statistical explanation of the entropy is remained as an open question.

For the Einstein gravity, a statistical derivation of the Bekenstein-Hawking entropy has been carried out [4] [5] for a black hole in three dimensional anti-de Sitter spacetime $\left(A d S_{3}\right)$ which is called the BTZ black hole [6] [7] [8]. Although several issues remain open to be resolved [9], this is one of the important examples which demonstrate the equality between the Bekenstein-Hawking entropy and a statistical entropy. It is our central interest to give a statistical interpretation of the black hole entropy in the diffeomorphism invariant theories of gravity. In this paper, we attempt to derive the statistical entropy of the BTZ black hole in the higher curvature gravity.

The computation of the statistical entropy in refs. 消 and [5] makes use of the correspondence between the asymptotic symmetry of the BTZ black hole spacetime and the conformal field theory (CFT) constructed on the boundary of the spacetime, which is called the AdS/CFT correspondence [10] [11]. The Virasoro algebra satisfied by the generators of the CFT includes a central charge, which, in the Einstein gravity, can be calculated by making use of the Hamilton formalism of gravity. Thus, when we extend the entropy computation to the higher curvature gravity, it becomes full of intricacies to construct Hamiltonian. To avoid this complexity, we transform the original frame of the higher curvature gravity by defining a new metric into the Einstein frame where the new metric obeys the Einstein-Hilbert action and an auxiliary matter field appears. Then the central charge can be calculated in the Einstein frame to

\footnotetext{
${ }^{\dagger}$ E-mail: saida@phys.h.kyoto-u.ac.jp

${ }^{\ddagger}$ E-mail: jiro@phys.h.kyoto-u.ac.jp
} 
give the statistical entropy of the BTZ black hole in the higher curvature gravity. It is shown that the statistical entropy is equal to the geometrical one of eq. (1) in the higher curvature gravity. Although our results in this paper is restricted to the three dimensional higher curvature gravity, it suggests that the equivalence between the geometrical and statistical entropies is retained in the general diffeomorphism invariant theories of gravity.

In section II we review the BTZ black hole and the statistical derivation of its entropy in the Einstein gravity. Section III is devoted to the computation of the BTZ black hole entropy in the higher curvature gravity, and our results are illustrated with an example of the Lagrangian $R+a R^{2}+b R_{\mu \nu} R^{\mu \nu}-2 \Lambda$. Finally we give summary and discussion in section [V, where the implication of our results will be discussed. Throughout this paper we use the unit, $c=\hbar=1$.

\section{BTZ BLACK HOLE ENTROPY IN THE EINSTEIN GRAVITY}

For the preparation of our purpose, in this section, we review the BTZ black hole [6] [7] [8] and its statistical entropy in the Einstein gravity [5]. The action we treat here is

$$
I=\frac{1}{16 \pi G} \int d^{3} x(R-2 \Lambda) \sqrt{-g}+B,
$$

where $\Lambda$ is the cosmological constant and $B$ is a surface term which is needed to let the variation of $I$ make sense [6].

\section{A. BTZ Black Hole}

Because three dimensional gravity have no dynamical degrees of freedom, a black hole spacetime is locally equivalent to $A d S_{3}$. Then it is enough for our purpose to consider only a constant negative curvature spacetime. The Riemann tensor of the constant curvature spacetime is expressed as

$$
R_{\mu \nu \alpha \beta}=\frac{R}{6}\left(g_{\mu \alpha} g_{\nu \beta}-g_{\mu \nu} g_{\alpha \beta}\right),
$$

where $R$ is the Ricci scalar which is related to the cosmological constant through the Einstein equation: $R=6 \Lambda$. Further we can set $R=-6 / l^{2}$ where $l$ is the curvature radius of $A d S_{3}$. Note that $l$ is related to the cosmological constant, $\Lambda=-6 / l^{2}$.

The BTZ black hole spacetime can be constructed by manipulating geometrically the global $A d S_{3}$ [6], here the global $A d S_{3}$ denotes the $A d S_{3}$ spacetime without any source in it. This manipulation is a discrete identification of points in the global $A d S_{3}$ along one Killing vector. It is convenient for describing this identification to embed the global $A d S_{3}$ into the four dimensional flat space of metric signature $(-,-,+,+)$. From this viewpoint, the global $A d S_{3}$ is given by

$$
d s^{2}=-d u^{2}-d v^{2}+d x^{2}+d y^{2} \quad \text { with }-u^{2}-v^{2}+x^{2}+y^{2}=-l^{2}
$$

where $l$ is the curvature radius of the global $A d S_{3}$. The construction of the BTZ black hole of mass $M$ and angular momentum $J$ is accomplished by identifying discretely the points in the global $A d S_{3}$ with the exponential mapping:

$$
p \rightarrow \exp (2 \pi m \zeta) p \text { for } p \in A d S_{3},
$$

where $m=0, \pm 1, \pm 2, \cdots$ and $\zeta$ is the Killing vector given by

$$
\zeta=\frac{r_{+}}{l}\left(x \partial_{u}+u \partial_{x}\right)-\frac{r_{-}}{l}\left(y \partial_{v}+v \partial_{y}\right) \quad, \quad r_{ \pm}=\sqrt{2 G l(M+J)} \pm \sqrt{2 G l(M-J)},
$$

where we use non-standard convention that $M$ and $J$ have no dimension. By giving the following coordinate transformation from $(u, v, x, y)$ to $(t, r, \phi)$ makes the discrete identification be more understandable:

$$
(u, v, x, y)=(\sqrt{A} \cosh Z, \sqrt{B} \sinh T, \sqrt{A} \sinh Z, \sqrt{B} \cosh T),
$$

where $A(r)=l^{2}\left(r^{2}-r_{-}^{2}\right) /\left(r_{+}^{2}-r_{-}^{2}\right), B(r)_{c}=l^{2}\left(r^{2}-r_{+}^{2}\right) /\left(r_{+}^{2}-r_{-}^{2}\right), T(t, \phi)=l^{-1}\left[\left(r_{+} / l\right) t-r_{-} \phi\right], Z(t, \phi)=$ $l^{-1}\left[-\left(r_{-} / l\right) t+r_{+} \phi\right]$ and $r_{ \pm}$is given in eq. (6). By this transformation, the metric (4) becomes 


$$
d s^{2}=-N^{2} d t^{2}+\frac{1}{N^{2}} d r^{2}+r^{2}\left[d \phi+N^{\phi} d t\right]^{2},
$$

where $-\infty<\phi<+\infty, N^{2}=(r / l)^{2}+(4 G J / r)^{2}-G M / l, N^{\phi}=-4 G J / r^{2}$. This metric is not of black hole spacetime, unless the coordinate $\phi$ is periodic as an angular coordinate. On the other hand, by this transformation, the Killing vector $\zeta$ becomes $\zeta=\partial_{\phi}$. Thus the discrete identification (5) makes the coordinate $\phi$ be periodic, $\phi \sim \phi+2 \pi$. That is, the BTZ black hole is constructed from the global $A d S_{3}$ by the discrete identification mentioned above, where $M$ and $J$ are the mass and the angular momentum of the BTZ black hole, respectively. The event horizon is at $r_{+}$, further the Bekenstein-Hawking entropy is calculated to be

$$
S_{B H}=\frac{1}{4 G} \oint_{r_{+}} d \phi \sqrt{g_{\phi \phi}}=\frac{\pi}{4 G}[\sqrt{8 G l(M+J)}+\sqrt{8 G l(M-J)}] .
$$

\section{B. Statistical Entropy}

The BTZ black hole entropy can be calculated by counting the number of states [5] [9]. The counted states are in the Hilbert space operated by the quantum counterparts (generators) of asymptotic symmetry of asymptotically $A d S_{3}$, whose definitions are described below [ [ 1 . The asymptotically $A d S_{3}$ metrics are defined as:

$$
\begin{gathered}
d s^{2}=\left[-\frac{r^{2}}{l^{2}}+O(1)\right] d t^{2}+\left[\frac{l^{2}}{r^{2}}+O\left(\frac{1}{r^{4}}\right)\right] d r^{2}+\left[r^{2}+O(1)\right] d \phi^{2} \\
+O\left(\frac{1}{r^{3}}\right) d t d r+O(1) d t d \phi+O\left(\frac{1}{r^{3}}\right) d r d \phi
\end{gathered}
$$

where $0<\phi \leq 2 \pi$. The global $A d S_{3}$ is included in these metrics which asymptote to $A d S_{3}$ as $r \rightarrow \infty$. It is obvious that the BTZ black hole is of asymptotically $A d S_{3}$. The asymptotic symmetry of asymptotically $A d S_{3}$ is described by Killing vectors, $\xi$, which preserve the boundary condition of the metric are expressed explicitly as 牛 河

$$
\begin{aligned}
& \xi^{t}=l\left(T^{+}+T^{-}\right)+\frac{l^{3}}{2 r^{2}}\left(\partial_{+}^{2} T^{+}+\partial_{-}^{2} T^{-}\right)+O\left(\frac{1}{r^{4}}\right), \\
& \xi^{r}=-r\left(\partial_{+} T^{+}+\partial_{-} T^{-}\right)+O\left(\frac{1}{r}\right), \\
& \xi^{\phi}=T^{+}-T^{-}-\frac{l^{2}}{2 r^{2}}\left(\partial_{+}^{2} T^{+}-\partial_{-}^{2} T^{-}\right)+O\left(\frac{1}{r^{4}}\right),
\end{aligned}
$$

where $T^{ \pm}=T^{ \pm}\left(x^{ \pm}\right)$and $x^{ \pm}=t / l \pm \phi$. The Killing vectors in this form include those of the global $A d S_{3}$.

The algebra satisfied by the quantum counterparts (generators) of asymptotic symmetry of asymptotically $A d S_{3}$ should be of the quantization of the classical algebra satisfied by classical generators of the symmetry, which is just the Hamiltonian generating the diffeomorphism along the Killing vector $\xi$ [4. Such a Hamiltonian is expressed as $H[\xi]=$ $\int_{\Sigma} d^{2} x \xi^{a} \mathcal{H}_{a}+Q[\xi]$, where $\Sigma$ is the spatial hypersurface, $\xi^{a}(a=\perp, 1,2)$ are the vertical and parallel components of $\xi$ with respect to $\Sigma, \mathcal{H}_{a}$ are the constraints and $Q[\xi]$ is the surface term which is needed to cancel the surface term in the variation $\delta H[\xi]$, that is, there is a freedom of additional constant to $Q$. Given two Hamiltonians, $H[\xi]$ and $H[\eta]$, the algebra of them is obtained by evaluating the Poisson bracket. It is shown in ref. [⿴囗十 that such an algebra is the central extension of the commutation relation (Lie bracket) of $\xi$ and $\eta,\{H[\xi], H[\eta]\}_{P}=H[\{\xi, \eta\}]+K[\xi, \eta]$, where $K[\xi, \eta]$ is the central extension, \{\}$_{P}$ is the Poisson bracket and $\{\xi, \eta\}$ is the Lie bracket. Further, because the constraints $\mathcal{H}_{a}$ is weakly zero, it reduces to the algebra: $\{Q[\xi], Q[\eta]\}_{D}=Q[\{\xi, \eta\}]+K[\xi, \eta]$, where \{\}$_{D}$ is the Dirac bracket. The central extension $K[\xi, \eta]$ can be calculated by setting the surface term $Q$ for the global $\operatorname{Ad} S_{3}$ be zero 画:

$$
K[\xi, \eta]=\lim _{r \rightarrow \infty} \frac{1}{16 \pi G} \oint d S_{l}\left\{\widehat{G}^{i j k l}\left[\xi^{\perp} g_{i j \mid k}-\xi_{, k}^{\perp}\left(g_{i j}-\hat{g}_{i j}\right)\right]+2\left(\xi^{i}+N^{i} \xi^{\perp}\right) \pi_{i}^{l}\right\}
$$

where the latin indices denote the spatial coordinates, $\hat{g}_{\mu \nu}$ is the global $A d S_{3}, g_{\mu \nu}$ is the asymptotically $A d S_{3}$ of the form 10), $\widehat{G}^{i j k l}=(1 / 2) \sqrt{\operatorname{det}\left(\hat{g}_{i j}\right)}\left(\hat{g}^{i k} \hat{g}^{j l}+\hat{g}^{i l} \hat{g}^{j k}-2 \hat{g}^{i j} \hat{g}^{k l}\right), d S_{l}$ is the line element of $r=$ const. circle on the spatial hypersurface $\Sigma, \pi_{i}^{l}=g^{k l} \pi_{i k}, \pi_{i j}$ is the conjugate momentum of $g_{i j}, A_{\mid k}$ is covariant derivative of quantity 
$A$ with respect to spatial metric $\hat{g}_{i j}, \xi^{\perp}=N^{\perp} \xi^{t}$, and $N^{\perp}$ and $N^{i}$ are the lapse function and the shift vector of $g_{\mu \nu}$ respectively. Here the variation of metric from $\hat{g}_{\mu \nu}$ to $g_{\mu \nu}$ is given by $\delta g_{\mu \nu} \equiv g_{\mu \nu}-\hat{g}_{\mu \nu}=\mathcal{L}_{\eta} \hat{g}_{\mu \nu}$.

When we define the Fourier components of $\xi$ as $\xi_{m}^{ \pm} \equiv(i / 2) \xi$ with $T^{ \pm}=\exp \left(i m x^{ \pm}\right)(m=0, \pm 1, \pm 2 \cdots)$, the Lie brackets of the Killing vectors are calculated to be the Virasoro algebra without central extension: $\left\{\xi_{m}^{ \pm}, \xi_{n}^{ \pm}\right\}=$ $(m-n) \xi_{m+n}^{ \pm},\left\{\xi_{m}^{+}, \xi_{n}^{-}\right\}=O(1 / r)$. Then the classical algebra we are seeking can be expressed as the Virasoro algebra with the central extension given by eq. (12). That is, the algebra of the quantum generators is also the same algebra, which is obtained by quantizing the commutation relation through the replacement, \{\}$_{D} \rightarrow-i[]$, where [] is the commutation relation of quantum operator [⿰亻弋 :

$$
\begin{aligned}
& {\left[L_{m}, L_{n}\right]=(m-n) L_{m+n}+\frac{C}{12} m\left(m^{2}-1\right) \delta_{m+n, 0},} \\
& {\left[\tilde{L}_{m}, \tilde{L}_{n}\right]=(m-n) \tilde{L}_{m+n}+\frac{C}{12} m\left(m^{2}-1\right) \delta_{m+n, 0},} \\
& {\left[L_{m}, \tilde{L}_{n}\right]=0 .}
\end{aligned}
$$

Here $L_{m}$ and $\tilde{L}_{m}$ denote the quantum generators corresponding to $\xi_{m}^{+}$and $\xi_{m}^{-}$respectively, and the central charge $C$ can be obtained by substituting the metric given by eq. (10) into $g_{\mu \nu}$ in eq. (12) to be a positive constant [4],

$$
C=\frac{3 l}{2 G} .
$$

Thus the quantum gravity of $A d S_{3}$ is related to the conformal field theory (CFT) on the boundary of the spacetime. Such a correspondence between the $A d S$ and the CFT is called the AdS/CFT correspondence [10] [11].

With above preparations, we can proceed to a calculation of statistical entropy. The calculation follows the ordinary state counting of the conformal field theory, where the counted states are the eigen states of $L_{0}$ and $\tilde{L}_{0}$. For the case that the eigen values are large (semi-classical), we can obtain the number of states by Cardy's formula [9] [12] to give the statistical entropy:

$$
S_{C} \approx 2 \pi \sqrt{\frac{C \lambda}{6}}+2 \pi \sqrt{\frac{C \tilde{\lambda}}{6}},
$$

where $\lambda$ and $\tilde{\lambda}$ are the (large) eigen values of $L_{0}$ and $\tilde{L}_{0}$ respectively.

Note that $\xi_{0}^{+}+\xi_{0}^{-}=2 l \partial_{t}+O\left(1 / r^{4}\right)$ and $\xi_{0}^{+}-\xi_{0}^{-}=2 \partial_{\phi}+O\left(1 / r^{4}\right)$, then a mass operator $\mathcal{M}$ and an angular momentum operator $\mathcal{J}$ are defined by $\mathcal{M} \equiv L_{0}+\tilde{L}_{0}$ and $\mathcal{J} \equiv L_{0}-\tilde{L}_{0}$, respectively [5]. For the BTZ black hole, we define a quantum state $|M J\rangle$ by $\mathcal{M}|M J\rangle=M|M J\rangle$ and $\mathcal{J}|M J\rangle=J|M J\rangle$, further the ground state by $\mathcal{M}|00\rangle=0$ and $\mathcal{J}|00\rangle=0$, that is, $M=J=0$. The spacetime of the ground state asymptotes to the $A d S_{3}$ as $r \rightarrow \infty$, thus this ground state can correspond to $\hat{g}_{\mu \nu}$ in eq. (12). Consequently, the above calculations of the central charge and the statistical entropy are available for the BTZ black hole [9]. The state, $|M J\rangle$, provides the eigen values of $L_{0}$ and $\tilde{L}_{0}$ to be

$$
\lambda=\frac{1}{2}(M+J) \quad, \quad \tilde{\lambda}=\frac{1}{2}(M-J),
$$

then the statistical entropy for the case of large $M$ and $J$ becomes

$$
S_{C}=\frac{\pi}{4 G}[\sqrt{8 G l(M+J)}+\sqrt{8 G l(M-J)}] .
$$

This coincides with the Bekenstein-Hawking entropy $S_{B H}$ given by eq. (9).

\section{BTZ BLACK HOLE ENTROPY IN THE HIGHER CURVATURE GRAVITY}

In this section, we calculate the statistical entropy of the BTZ black hole in the higher curvature gravity. The action we treat here is of the form:

$$
I=\frac{1}{16 \pi G} \int d^{3} x f\left(R_{\mu \nu}, g^{\mu \nu}\right) \sqrt{-g}+B
$$


where $f\left(R_{\mu \nu}, g^{\mu \nu}\right)$ is the Lagrangian of general higher curvature gravity and $B$ is the surface term needed by the same reason as the action (2). This action is the most generic form of the three dimensional higher curvature gravity because the Weyl tensor vanishes in three dimensional spacetime, that is, the Riemann tensor can be expressed generally by the Ricci tensor, the Ricci scalar and the metric:

$$
R_{\mu \nu \alpha \beta}=g_{\mu \alpha} R_{\nu \beta}+g_{\nu \beta} R_{\mu \alpha}-g_{\nu \alpha} R_{\mu \beta}-g_{\mu \beta} R_{\nu \alpha}-\frac{1}{2}\left(g_{\mu \alpha} g_{\nu \beta}-g_{\mu \beta} g_{\nu \alpha}\right) R
$$

When we are interested in the statistical entropy of the BTZ black hole in the higher curvature gravity, the entropy is given by the same formula as eq. (15) with the central charge and the eigen values of the Virasoro generators in the higher curvature gravity. The eigen values (16) can be easily obtained through the Noether charge form of the mass and the angular momentum constructed in ref. [1]. However, as reviewed in the previous section, the central charge is necessary to calculate the surface terms of the Hamiltonian, which should be corrected by the higher curvature terms in the Lagrangian to induce additional terms upon the central charge, eq. (12). The derivation and resultant form of the additional terms will be very complicated. To avoid this intricacy, we define new metric and transform the original higher curvature frame into the Einstein frame.

\section{A. Frame Transformation}

In this subsection, we review the transformation from the original higher curvature frame to the Einstein frame in preparation for calculating the central charge. The Euler-Lagrange equation in the original frame is obtained as

$$
\frac{\partial f}{\partial g^{\mu \nu}}-\frac{1}{2} f g_{\mu \nu}=\frac{1}{2}\left[P_{\alpha \mu ; \nu}^{; \alpha}+P_{\alpha \nu ; \mu}^{; \alpha}-\square P_{\mu \nu}-g_{\mu \nu} P_{\alpha \beta}^{; \alpha \beta}\right],
$$

where $P_{\mu \nu} \equiv g_{\mu \alpha} g_{\nu \beta}\left(\partial f / \partial R_{\alpha \beta}\right)$. This is obviously a fourth order differential equation of $g^{\mu \nu}$. The new metric which turns out to be that in the Einstein frame is defined by [13] [14]

$$
\bar{g}^{\mu \nu} \equiv\left[-\operatorname{det}\left(\frac{\partial(f \sqrt{-g})}{\partial R_{\alpha \beta}}\right)\right]^{-1} \frac{\partial(f \sqrt{-g})}{\partial R_{\mu \nu}} .
$$

We assume here that the relation (21) can be inverted to give $R_{\mu \nu}$ as a function of $\bar{g}^{\alpha \beta}$ and $g^{\alpha \beta}$ (including no derivative of them):

$$
R_{\mu \nu}=R_{\mu \nu}\left(\bar{g}^{\alpha \beta}, g^{\alpha \beta}\right)
$$

The alternative action which is equivalent to the original action $I$ and describes the Einstein frame can be defined by

$$
\begin{aligned}
\bar{I} \equiv \frac{1}{16 \pi G} \int d^{3} x \sqrt{-\bar{g}}\left[\bar{g}^{\mu \nu} R_{\mu \nu}\left(g^{\alpha \beta}, \partial_{\omega} g^{\alpha \beta}, \partial_{\tau} \partial_{\omega} g^{\alpha \beta}\right)\right. \\
\left.-\bar{g}^{\mu \nu} R_{\mu \nu}\left(\bar{g}^{\alpha \beta}, g^{\alpha \beta}\right)+\frac{\sqrt{-g}}{\sqrt{-\bar{g}}} f\left(R_{\mu \nu}\left(\bar{g}^{\alpha \beta}, g^{\alpha \beta}\right), g^{\mu \nu}\right)\right]+\bar{B} .
\end{aligned}
$$

Note that $\bar{g}^{\mu \nu}$ and $g^{\mu \nu}$ are treated as independent dynamical variables in this action. It can be easily checked that the Euler-Lagrange equations of $\bar{I}$ are equivalent to those of $I$. That is, $\bar{I}$ is a well-defined Lagrangian which is equivalent to $I$.

In order to understand that the alternative action $\bar{I}$ describes the Einstein frame, we rewrite $\bar{I}$ to an explicit form consisting of the Einstein-Hilbert action of $\bar{g}^{\mu \nu}$ and an auxiliary matter field. The following general relation is useful for such a purpose:

$$
\begin{aligned}
R_{\mu \nu}\left(g^{\alpha \beta}, \partial_{\omega} g^{\alpha \beta}, \partial_{\tau} \partial_{\omega} g^{\alpha \beta}\right)= & \bar{R}_{\mu \nu}\left(\bar{g}^{\alpha \beta}, \partial_{\omega} \bar{g}^{\alpha \beta}, \partial_{\tau} \partial_{\omega} \bar{g}^{\alpha \beta}\right) \\
& +\bar{\nabla}_{\alpha} F_{\mu \nu}^{\alpha}-\bar{\nabla}_{\nu} F_{\mu \alpha}^{\alpha}+F_{\alpha \beta}^{\alpha} F_{\mu \nu}^{\beta}-F_{\mu \alpha}^{\alpha} F_{\alpha \nu}^{\beta},
\end{aligned}
$$

where $\bar{\nabla}_{\mu}$ is the covariant derivative with respect to $\bar{g}^{\mu \nu}$ and $F_{\mu \nu}^{\alpha}$ is defined by

$$
F_{\mu \nu}^{\alpha} \equiv \Gamma_{\mu \nu}^{\alpha}-\bar{\Gamma}_{\mu \nu}^{\alpha}=\frac{1}{2} g^{\alpha \beta}\left(\bar{\nabla}_{\nu} g_{\mu \beta}+\bar{\nabla}_{\mu} g_{\beta \nu}-\bar{\nabla}_{\beta} g_{\mu \nu}\right)
$$

By substituting this relation into $\bar{I}$, we obtain 


$$
\begin{array}{r}
\bar{I}=\frac{1}{16 \pi G} \int d^{3} x \sqrt{-\bar{g}}\left[\bar{g}^{\mu \nu} \bar{R}_{\mu \nu}\left(\bar{g}^{\alpha \beta}, \partial_{\omega} \bar{g}^{\alpha \beta}, \partial_{\tau} \partial_{\omega} \bar{g}^{\alpha \beta}\right)+\bar{g}^{\mu \nu}\left(F_{\alpha \beta}^{\alpha} F_{\mu \nu}^{\beta}-F_{\mu \alpha}^{\alpha} F_{\alpha \nu}^{\beta}\right)\right. \\
\left.-\bar{g}^{\mu \nu} R_{\mu \nu}\left(\bar{g}^{\alpha \beta}, g^{\alpha \beta}\right)+\frac{\sqrt{-g}}{\sqrt{-\bar{g}}} f\left(R_{\mu \nu}\left(\bar{g}^{\alpha \beta}, g^{\alpha \beta}\right), g^{\mu \nu}\right)\right]+\bar{B}^{\prime},
\end{array}
$$

where $\bar{B}^{\prime}$ is different from $\bar{B}$ by a surface term corresponding to that including second derivatives of $g^{\mu \nu}$ in the right-hand side of eq. (24). The Einstein frame described by $\bar{I}$ is understood as the system consisting of the Einstein gravity, $\bar{g}^{\mu \nu}$, and an auxiliary tensor matter field, $g^{\mu \nu}$.

\section{B. Central charge in the higher curvature gravity}

We restrict our treatment hereafter to the case that the right hand side of the Euler-Lagrange equation (20) vanishes, that is, the spacetime is of the constant curvature. Further we assume that the curvature $R$ is of negative. Under such conditions, the BTZ black hole of the form given by eq. (8) can exist. Because the spacetime of the BTZ black hole is of constant curvature, the following quantity $\Omega$ is constant:

$$
\Omega=\frac{1}{3} g^{\mu \nu} \frac{\partial f}{\partial R_{\mu \nu}} .
$$

Eq. (21) gives the metric in the Einstein frame $\bar{g}^{\mu \nu}$ to be

$$
\bar{g}^{\mu \nu}=\Omega^{-2} g^{\mu \nu} \quad, \quad \bar{g}_{\mu \nu}=\Omega^{2} g_{\mu \nu},
$$

where $\bar{g}_{\mu \nu}$ is defined by $\bar{g}_{\mu \alpha} \bar{g}^{\alpha \nu}=\delta_{\mu}^{\nu}$. This is just a conformal transformation with constant conformal factor $\Omega$. The calculation of the central charge in the Einstein frame can be carried out by relating the quantities in the Einstein

frame to those in the original frame through the conformal transformation (28). Note that, because the isometries of the BTZ black hole in both frames are the same, the asymptotic symmetry Killing vectors (11) in both frames are the same. Further by definitions of $\widehat{G}^{i j k l}, \pi_{i}^{l}$, the lapse function and the shift vector, we can extract their relations between both frames. These relations are obtained as

$$
\bar{\xi}^{\mu}=\xi^{\mu} \quad, \quad \overline{\widehat{G}}^{i j k l}=\Omega^{-2} \widehat{G}^{i j k l} \quad, \quad \bar{\pi}_{i}^{l}=\Omega \pi_{i}^{l} \quad, \quad \bar{N}^{\perp}=\Omega N^{\perp} \quad, \quad \bar{N}^{i}=\Omega^{2} N^{i} .
$$

Substituting these results into (12), the central charge is calculated to be

$$
C=\frac{l}{2 G} g^{\mu \nu} \frac{\partial f}{\partial R_{\mu \nu}} .
$$

Although this is the central charge calculated in the Einstein frame, this central charge is equivalent to that in the original higher curvature frame.

\section{Computation of the BTZ black hole entropy}

With all the preparations done above, we can proceed to the calculation of the BTZ black hole entropy in the higher curvature gravity. The BTZ black hole is also of the asymptotically $A d S_{3}$ spacetime even in the higher curvature gravity. Hence the AdS/CFT correspondence is available 10 11] to give the statistical entropy of the form (15). When we denote the BTZ black hole metric in the higher curvature gravity by the same notation as eq. (8), the mass and the angular momentum in the higher curvature gravity are calculated through the Noether charge form obtained in ref. [1] to be $\Omega M$ and $\Omega J$, respectively. Then the two Virasoro eigen values $\lambda$ and $\tilde{\lambda}$ in the higher curvature gravity are given by the same procedure as eq. (16),

$$
\lambda=\Omega \frac{1}{2}(M+J) \quad, \quad \tilde{\lambda}=\Omega \frac{1}{2}(M-J) .
$$

Finally the statistical entropy of the BTZ black hole in the higher curvature gravity is obtained by eqs. (15), (30) and (31) as: 


$$
\begin{aligned}
S_{C} & =\Omega \frac{\pi}{4 G}[\sqrt{8 G l(M+J)}+\sqrt{8 G l(M-J)}] \\
& =\frac{\pi}{12 G} g^{\mu \nu} \frac{\partial f}{\partial R_{\mu \nu}}[\sqrt{8 G l(M+J)}+\sqrt{8 G l(M-J)}]
\end{aligned}
$$

On the other hand, the geometrical entropy of the black hole can be calculated by eq. (1) to be

$$
S_{I W}=-\frac{1}{8 G} \oint_{H} d \phi \sqrt{g_{\phi \phi}} \frac{\partial f}{\partial R_{\mu \alpha}} g^{\nu \beta} \epsilon_{\mu \nu} \epsilon_{\alpha \beta}=\frac{1}{12 G} g^{\mu \nu} \frac{\partial f}{\partial R_{\mu \nu}} \oint_{r_{+}} d \phi \sqrt{g_{\phi \phi}},
$$

By making use of the integral in (9), this formula (33) coincides with the statistical entropy $S_{C}$ given by eq. (32).

$$
\text { D. Example: } f=R+a R^{2}+b R_{\mu \nu} R^{\mu \nu}-2 \Lambda
$$

We apply the results obtained above to the case,

$$
f=R+a R^{2}+b R_{\mu \nu} R^{\mu \nu}-2 \Lambda,
$$

where $a$ and $b$ are constants. For the constant curvature spacetime, the Euler-Lagrange equation (20) becomes

$$
(1+2 a R) R_{\mu \nu}+\frac{1}{2}\left(R+a R^{2}+b R_{\alpha \beta} R^{\alpha \beta}-2 \Lambda\right) g_{\mu \nu}=0 .
$$

For the BTZ black hole which is the negative constant curvature space, $\Lambda$ is related to the Ricci scalar through eqs. (3) and (35) as

$$
\Lambda=\frac{R}{6}[1+(b-a) R] .
$$

The conformal transformation 28 becomes

$$
\bar{g}_{\mu \nu}=\Omega^{2} g_{\mu \nu} \quad, \quad \Omega=1-\frac{12 a+4 b}{l^{2}},
$$

where we have used the definition of the radius of $A d S$ spacetime: $l=\sqrt{-6 / R}$. This gives the central charge through eq. (30) as

$$
C=\left(1-\frac{12 a+4 b}{l^{2}}\right) \frac{3 l}{2 G},
$$

which is consistent with the results obtained in ref. [11]. We obtain the statistical entropy in the higher curvature gravity through eq. (32)

$$
S_{C}=\left(1-\frac{12 a+4 b}{l^{2}}\right) \frac{\pi}{4 G}[\sqrt{8 G l(M+J)}+\sqrt{8 G l(M-J)}] .
$$

The geometrical entropy given by eq. (II) is calculated to be

$$
S_{I W}=\left(1-\frac{12 a+4 b}{l^{2}}\right) \frac{1}{4 G} \oint_{r_{+}} d \phi \sqrt{g_{\phi \phi}} .
$$

By making use of the integral in (9), this equation (40) coincides with the statistical entropy $S_{C}$ given by eq. (39).

\section{SUMMARY AND DISCUSSION}

We have shown the statistical derivation of the BTZ black hole entropy in the higher curvature gravity. The resultant formula agrees with the one derived by the Noether charge method [1]. As a by-product, we have obtained the central charge, that is, the coefficient of the Weyl anomaly, in the higher curvature gravity. Although the statistical entropy is calculated in the higher curvature gravity, because the procedure of our calculation is essentially the same 
as the Einstein gravity, some issues which stemmed from the use of CFT on the boundary of $A d S_{3}$ [9] remain open to be resolved.

Provided that, instead of the higher curvature gravity, we adopt the BTZ black hole in diffeomorphism invariant theories of gravity which include symmetrized covariant derivatives of Riemann tensor like $R_{\mu \nu \alpha \beta ;(\omega \tau)}$, the problem which arises in applying our procedure to calculate the central charge is that we do not have the formalism of transforming the frames in such theories. However, because the relation (3) denotes that the terms of covariant derivative of Riemann tensor in such Lagrangians vanishes, it is expected that the frame transformation between the original and Einstein frames is also the conformal transformation with constant conformal factor. For the other case of Lagrangians include some matter fields coupling to the gravity, the factor $\Omega$ should depend on the matter fields. However it is possible to set such matter fields be constant without loss of consistency with the BTZ black hole. Then our computation of the statistical entropy of the BTZ black hole in this paper is available for the general diffeomorphism invariant theories of gravity which include the symmetrized covariant derivatives of Riemann tensor and the matter fields coupling to the gravity, provided that we can construct a well-defined transformation of the frames in such theories. Thus, it is natural to conjecture that the equality between the geometrical entropy and the statistical one is retained in the general diffeomorphism invariant theories of gravity. Further, with noting the work 15] which extends the computation of the statistical entropy of the BTZ black hole to any dimension in the Einstein gravity, it is expected that the equivalence between the geometrical and statistical entropies can be extended to any dimensional diffeomorphism invariant theories of gravity.

The derivation of the statistical entropy in this paper is heavily relied on the conformal field theory constructed on the boundary of the BTZ black hole spacetime. It has been well recognized that the unitarity of the conformal field theory requires the positivity of the central charge, which is consistent with eq. (14). Further it is required through eq. (30) that $\Omega>0$. On the other hand, this relation, $\Omega>0$, is required from the null energy condition in the Einstein frame and the weak cosmic censorship conjecture [16] in considering the second law of the Black hole thermodynamics for the geometrical entropy as mentioned below [3]. Both of these conditions are the essential assumptions in establishing the second law in the Einstein gravity (the area theorem). For the case of the Lagrangian polynomial in $R$, it has already been explicitly derived in ref. [3] that the null energy condition in the Einstein frame requires the positivity of the conformal factor $\Omega$ of the frame transformation. This suggests that this requirement can be extended to the case of general higher curvature gravity. Further, with making use of the frame transforation in considering the second law in the diffeomorphism invariant theories of gravity, the cosmic censorship conjecture will be required to be satisfied in the Einstein frame. It will also be necessary to retain the disappearance of naked singularities in the original frame. Then we require the positivity of $\Omega$ because, for the case that $\Omega \leq 0$, there is a possibility of appearing singularities in the original frame. Thus, the cosmic censorship conjecture and the null energy condition in the Einstein frame must have some relation with the unitarity of the conformal field theory on the boundary of spacetime. The issue about the details of such a relation is left as an interesting open question.

We turn our attention to some possible applications of our results to some interesting cases. As we have already seen, the statistical black hole entropy can be computed by using the data on the boundary of the black hole spacetime which asymptotes to that of anti-de Sitter spacetime. This kind of correspondence between the symmetry of the boundary of $A d S$ spacetime and the conformal field theory (CFT) has been intensively studied recently. This correspondence can be used to deduce the entropy of the black string system in higher dimensions [17]. It is interesting to investigate whether or not our results of this paper can be extended to the higher dimensional black objects.

For the calculation of the statistical entropy through the AdS/CFT correspondence, the central importance exists in the correspondence between the symmetry of the boundary of $A d S$ and the isometry of the bulk spacetime of a black hole, such as the stationarity and axisymmetry. Hence, it is not obvious whether or not the statistical entropy which we have calculated can be extended to the dynamical cases such as the collapsing black holes and the evaporating black holes. As the geometrical entropy is extended with retaining its meaning even to the dynamical systems [1] [2], however, it is expected to be able to extend our results to the dynamical cases.

\section{ACKNOWLEDGEMENTS}

We would like to Thank M.Sakagami for his useful discussions. This work was supported in part by Monbusho Grant-in-Aid for Scientific Research No.10740118. 
[1] R.Wald, Phys.Rev.D48 R3427(1993); V.Iyer and R.Wald, Phys.Rev.D50 846(1994), Phys.Rev.D52 4430(1995)

[2] T.Jacobson, G.Kang and R.Myers, Phys.Rev.D49 3518(1994)

[3] T.Jacobson, G.Kang and R.Myers, Phys.Rev.D52 3518(1995)

[4] J.Brown and M.Henneaux, Commun.Math.Phys.104 207(1986)

[5] A.Strominger, J.High Energy Phys.9802 009(1998), hep-th/9712251

[6] M.Bañados, M.Henneaux, C.Teitelboim and J.Zanelli, Phys.Rev.D48 1506(1993)

[7] O.Coussaert and M.Henneaux, Phys.Rev.Lett72 183(1994)

[8] S.Carlip, Class.Quantum Grav.12 2853(1995)

[9] S.Carlip, Class.Quantum Grav.15 3609(1998)

[10] See for example: J.Maldacena, Adv.Theor.Math.Phys.2 231(1998); E.Witten, Adv.Theor.Math.Phys.2 505(1998); M.Henningson and K.Skenderis, J.High Energy Phys.9807 023(1998)

[11] S.Nojiri and S.Odintsov, hep-th/9903033

[12] J.Cardy, Nucl.Phys.B270 186(1986)

[13] G.Magnano, M.Ferraris, M.Francaviglia, General Rel.Grav.19 465(1987)

[14] G.Magnano, M.Ferraris, M.Francaviglia, Class.Quantum Grav.7 557(1990)

[15] S.Carlip, Phys.Rev.Lett.82 2828(1999)

[16] The null energy condition is expressed as $T_{\mu \nu} k^{\mu} k^{\nu} \geq 0$ for any null vector $k^{\mu}$. The weak cosmic cesorship conjecture means that any naked singularities never appear in the interesting dynamical processes.

[17] See for example: N.Kaloper, Phys.Lett.B434 285(1998); Y.Myung, N.Kim and H.Lee, Mod.Phys.Lett.A14 575(1999); N.Deger, A.Kaya, E.Sezgin and P.Sundell, hep-th/9908089 